\title{
Passgenau, vielfältig, individuell
}

\section{Erfolgsfaktoren der Freiwilligendienste in der Freien Wohlfahrtspflege}

\author{
RAINER HUB UND \\ MARTIN SCHULZE \\ Rainer Hub ist als Bundesreferent \\ bei der Diakonie Deutschland zu- \\ ständig für alle Formen des freiwilli- \\ gen Engagements einschließlich der \\ Freiwilligendienste. \\ www.diakonie.de
}

Martin Schulze ist Geschäftsführer der Evangelischen Freiwilligendienste, die als eine gGmbH alle nationalen und internationalen Freiwilligendienstprogramme im evangelischen Bereich bündelt. www.ev-freiwilligendienste.de

\author{
Die Freiwilligendienste in der Freien Wohlfahrtspflege \\ sind eine Erfolgsgeschichte. Am Beispiel der \\ Evangelischen Kirche und ihrer Diakonie kann \\ gezeigt werden, welche Faktoren für die \\ Vergangenheit bestimmend waren und worauf sich \\ die Freiwilligendienst künftig stützen können.
}

Ausgangspunkte für die Erfolgsgeschichte Diakonisches Jahr (DJ) und Freiwilliges Soziales Jahr (FSJ) war der Aufruf des Direktors der Diakonie Neuendettelsau: Er rief am 9. Mai 1954 die "weibliche evangelische Jugend « auf sich ein Jahr für die Diakonie zu engagieren. Das Diakonische Jahr war als Kind der Zivilgesellschaft geboren. Nach dem Motto »Back to the roots « wurde die Kampagne mit Gottesdienst und Festakt ebenfalls in Neuendettelsau Ende April dieses Jahres gestartet. In die Chronologie der sechs Jahrzehnte eingebettet waren Gespräche mit Zeitzeuginnen der ersten Stunde.

Nach dieser ersten Stunde zog die Idee schnell weite Kreise. Es folgten Aufrufe in anderen Landeskirchen. Ende der 1950er Jahre kann man von einem flächendeckenden Angebot in der evangelischen Kirche ausgehen. Auch in der ehemaligen DDR wurde das Konzept von kirchlichen Trägern aufgenommen und umgesetzt. Bald folgten die katholischen Kirche und andere Wohlfahrtsverbände. Die Konsequenz dieser dynamischen Entwicklung war 1964 die Verabschiedung des Bundesgesetzes zur Förderung des Freiwilligen Sozialen Jahres.

War der Ausgangspunkt für den Aufruf mitunter der Arbeitskräftemangel in den diakonischen Einrichtungen in der Nachkriegszeit, so wurde ein wesentlicher Erfolgsfaktor des Freiwilligen Sozialen Jahres schon damals implementiert: die pädagogische Begleitung der Freiwilligen und die Verknüpfung des praktischen Einsatzes mit Seminaren und Bildungseinheiten in einem Gesamtkonzept. Letzteres hat sich stets weiterentwickelt und wurde an sich wandelnde Situationen angepasst.

Das Diakonische Jahr und das Freiwillige Soziale Jahr in der Evangelischen Kirche ist eine Erfolgsgeschichte. Im ersten Jahr waren es 25 Freiwillige, die ihren Dienst begannen. Diese Zahl nahm in den folgenden Jahrzehnten kontinuierlich zu. 2006/2007 konnte das Erreichen von insgesamt 100.000 Freiwilligen seit 1954 gefeiert werden. Seitdem haben sich die jährlich Teilnehmenden weiter stark nach oben entwickelt. Seit 1954 haben inzwischen circa 170.000 Freiwillige einen Evangelischen Freiwilligendienst im Inland absolviert.

Mittlerweile engagieren sich rund 13.000 Freiwillige pro Jahr in einem Freiwilligendienst im Inland bei evangelischen Trägern und etwa 800 in einem Freiwilligendienst im Ausland in der Evangelischen Trägergruppe. Hinzu kommen weitere Anbieter von Auslandsdiensten im Raum der evangelischen Kirche.

Das Programm wird getragen von der Arbeitsgemeinschaft der Evangelischen Jugend und der Diakonie Deutschland. Jugendverband und Wohlfahrtsverband gestalten das Programm gemeinsam und entwickeln es weiter.

\section{Erfolgsfaktor Passgenauigkeit}

Das Freiwillige Soziale Jahr ist für die Freiwilligen ein attraktives Angebot. Viele suchen zwischen Schule und weiterer Ausbildung eine Phase der Orientierung für ihren weiteren Lebensweg. Dieser Wunsch nach einer Auszeit hat 
im Zuge der verdichteten Schulzeit (G8) und der verkürzten und verschulten Studiengänge in den letzten Jahren nochmal an Gewicht gewonnen.

Freiwilligendienste sind hier ein ideales Angebot. Die Freiwilligen können sich entsprechend ihrer Interessen persönlich weiterentwickeln. So nehmen sie viele Impulse mit. Der individuellen Vermittlung und Begleitung kommt dabei eine große Bedeutung zu.

\section{Erfolgsfaktor Vielfalt und Weiterentwicklung}

Die Freiwilligendienste sind deshalb so erfolgreich, weil sie sich auch bei den Evangelischen Trägern immer weiterentwickelt haben; auch mit Blick auf die Einsatzstellen. Interessierte können aus einer Vielzahl von Einsatzstellen mit sehr unterschiedlichen inhaltlichen Schwerpunkten auswählen. Neben den weiterhin starken Bereichen der Arbeit mit älteren Menschen, kranken Menschen, Menschen mit Behinderungen, gibt es verstärkt Angebote auch in Schulen, in Kirchengemeinden, in der Jugendarbeit
Aus Sicht von Diakonie und Evangelischer Jugend müssen sich die Freiwilligendienste aufbauend auf den äußerst positiven Erfahrungen der vergangenen Jahrzehnte auch weiterhin im Sinne des Subsidiaritätsprinzips weiterentwickeln:

\section{Erfolgsfaktor Trägerprinzip}

Der zentrale Erfolgsfaktor ist das Trägerprinzip. Die Träger stellen mit den Begleitkonzepten sicher, dass Freiwillige und Einsatzstellen eine unabhängige dritte Stelle haben, an die sie sich wenden können und die die Gesamtverantwortung für die Ausgestaltung des Dienstes inklusive der pädagogischen Begleitung tragen.

Derzeit gibt es 45 evangelische Träger, die einen Freiwilligendienst im Inland anbieten. 23 Träger sind angeschlossen die Dienste im Ausland anbieten. Dabei sind die Größen der Träger im Inland (zwischen 20 und 1800 Freiwilligen) und im Ausland (zwischen 5 und 220 Freiwilligen) sehr unterschiedlich. Manche arbeiten regional, andere agieren bundesweit.

\section{"Mitarbeitende aus Freiwilligendiensten bereichern und qualifizieren die Arbeit in Diensten und Einrichtungen der Freien Wohlfahrtspflege"}

und weiteren sozialen Feldern. Die deutliche Ausweitung von Einsatzmöglichkeiten im Ausland in den letzten Jahren trägt zudem zur Attraktivität der Freiwilligendienste bei.

Die unterschiedlichen Profile von Evangelischen Trägern tragen darüber hinaus dazu bei, dass Interessierte mit unterschiedlichen persönlichen Hintergründen für sie passende Begleit- und Einsatzkonzepte vorfinden. In den letzten Jahren wurde eine Vielzahl von Angeboten für Freiwillige mit besonderem Förderbedarf entwickelt und erprobt (z. B. »FSJ plus« des Diakonischen Werkes Württemberg, das »FSJ4You « des Diakonischen Werkes Hamburg oder das »FSJ fifty-fifty« des Diakonischen Werkes Schleswig-Holstein). Einige dieser Initiativen laufen bereits über Jahre und tragen somit dauerhaft zur Vielfalt der Angebote bei.
Diese Vielfalt stellt die Trägergruppe zwar immer wieder vor große Herausforderungen, sie bringt aber auch unterschiedliche Angebotsformen und Ansätze mit sich und eine Pluralität der Angebote, aus denen Freiwilligen auswählen können.

\section{Erfolgsfaktor Bildung und Qualität}

Die Träger stellen ebenso sicher, dass es ein an den Interessen der Freiwilligen ausgerichtetes ganzheitliches Bildungskonzept gibt. Dieses reicht vom Bewerbungsprozess über die persönliche Begleitung durch die Einsatzstellen und die Träger bis hin zu den Seminaren. Dabei ist die Partizipation der Freiwilligen bei der Ausgestaltung der Seminare und Angebote wesentlich.

Neben der persönlichkeitsbezogenen und sozialen Bildung, der interkulturel- len und politischen Bildung sowie der arbeitsweltlichen Bildung und beruflichen Orientierung ist bei den Evangelischen Freiwilligendiensten als konfessionellem Träger auch die religiöse Bildung eine wichtige Säule der Begleitung.

Die Evangelische Trägergruppe ist dabei offen für alle interessierten Menschen, unabhängig von konfessioneller oder religiöser Zugehörigkeit. Es steht vielmehr die Motivation der Interessierten im Vordergrund. Notwendig hierfür ist zugleich eine Offenheit der Freiwilligen, sich auf einen kirchlichen Träger und seine Werthaltungen einzulassen und sich mit beidem - auch kritisch - auseinanderzusetzen. Eine solche Offenheit für alle Interessierten bedeutet auch eine stetige Herausforderung für die Träger und die Gestaltung ihrer Programme.

Ausgangspunkt für die Arbeit der Trägergruppe sind die gemeinsame Bundeskonzeption und ein umfassendes Qualitätshandbuch mit Standards für die Durchführung der Dienste und deren kontinuierliche Weiterentwicklung. Solch eine Qualitätsentwicklung spielt seit Anfang und Mitte der 1990er Jahre eine wichtige Rolle. Gemeinsam mit den Trägern wurden in einem aufwendigen Prozess gemeinsame Standards für die Durchführung entwickelt. Klar definiert ist darin, wie die Begleitung der Freiwilligen und die Arbeit mit Einsatzstellen gestaltet werden soll. Auch welche Anforderungen an die Qualifikation der Mitarbeitenden und die Personalausstattung der Träger gestellt werden, ist bei der Arbeit der Qualitätsentwicklungskommission bedeutsam.

\section{Erfolgsfaktor Bedarf}

Schon die genannten Ursprünge des Diakonischen Werkes und des Freiwilligen Sozialen Jahres machen deutlich, dass die Einrichtung der Dienste eine Reaktion auch auf gesellschaftliche Bedarfe war. Auch heute haben Einsatzstellen ein Interesse mit Freiwilligen Mitarbeitende auf Zeit zu gewinnen, die die Arbeit zusätzlich qualifizieren und bereichern.

Neben der Deckung aktueller Bedarfe geht es aber insbesondere darum, Menschen für die Aufgaben im sozialen Bereich zu sensibilisieren. Das praktische Erleben dieser Handlungsfelder trägt bei einer Vielzahl der Freiwilligen auch dazu bei, dass sie im Anschluss hauptamtlich 


\section{Thesen zur Weiterentwicklung der Freiwilligendienste}

1 Für alle Interessierten existiert ein vielfäl- tiges und ausreichendes Angebot an Einsatzmöglichkeiten in Freiwilligendiensten.

In der Gesellschaft wird das Engage- ment der Freiwilligen auf allen Ebenen wertgeschätzt.

3 Freiwilligendienste sind in der Kirche, 3 . der Diakonie und in der Gesellschaft als attraktive Bildungs- und Orientierungszeiten anerkannt.

\section{Freiwilligendienste sind \\ F.inklusiv.}

Evangelische Freiwilligendienste sind . offen für alle Menschen.

Das evangelische Profil unserer Frei- willigendienste ist in der begleitenden Bildungsarbeit spürbar.

7 Bei sich verändernden demographischen - Verhältnissen bleiben die absoluten Zahlen an Freiwilligendienstleistenden stabil.

Freiwilligendienste werden von Män- nern und Frauen unabhängig ihres Geschlechts gleichermaßen geleistet.

arbeiten oder sich freiwillig in diesem Bereich weiter engagieren. Vor dem Hintergrund des demographischen Wandels ist dies auch eine wichtige Funktion der Freiwilligendienste.

Auch Menschen, die im Anschluss einen anderen beruflichen Weg einschlagen, nehmen die gesammelten Eindrücke und Erfahrungen im sozialen Bereich mit auf den weiteren Lebensweg und behalten - hoffentlich - ein Gespür für diesen für das gesellschaftliche Miteinander wichtigen Bereich.

\section{Wünsche und Erwartungen}

Die Freiwilligendienste im In- und Ausland waren in den letzten Jahren erheblichen Veränderungen unterworfen. Als Beispiele seien nach Aussetzung von Wehrpflicht und Zivildienst die Einführung des Bundesfreiwilligendienstes verbunden mit einem Ausbau der Freiwilligendienste sowie der Öffnung für andere Altersgruppen, die Einführung von weltwärts und des Internationalen Jugendfreiwilligendienstes, genannt. Hinzu kamen die Modellprojekte »Generationsübergreifende Freiwilligendienste « und »Freiwilligendienste aller
Freiwilligendienste haben ein interna- tionales Profil.

Vereinbarkeit von Familie, (Aus-) Bildung/Beruf, Engagement sowie individueller Lebensplanung ist auch ein Anspruch an Freiwilligendienste.

11 Freiwilligendienste unterliegen verbindlichen Qualitätsstandards.

12 Freiwilligendienste werden haupt• sächlich von Jugendlichen und jungen Erwachsenen geleistet, darüber hinaus gibt es zunehmend Freiwillige über 27 Jahre.

13 Freiwilligendienste grenzen sich . sowohl von anderen Formen Bürgerschaftlichen Engagements als auch von arbeitsmarktpolitischen Maßnahmen ab.

1 Freiwilligendienste werden unter - Sicherstellung des Subsidiaritätsprinzip von zivilgesellschaftlichen freien Trägern durchgeführt.

Quelle: "Evangelische Freiwilligendienste 2020". Nachzulesen sind die 14 Thesen ausführlicher unter www.diakonie.de.

Generationen« sowie der besondere Fokus auf bestimmte Zielgruppen (z. B. Freiwillige mit besonderem Förderbedarf).

All dies hat erhebliche quantitative und formale Veränderungen in die Arbeit der Träger gebracht. Benötigt wird daher eine Phase der Stabilität (auch der Förderpolitik) und der qualitativen Konsolidierung, in der die Programme auf Grundlage der gemachten Erfahrungen in der Form und Durchführung dergestalt angepasst werden, dass die Träger sich wieder auf die Arbeit mit den Freiwilligen und Einsatzstellen konzentrieren können.

Zentrale Überlegungen der Evangelischen Trägergruppe sind dabei die Verringerung des bürokratischen Aufwandes und die Fokussierung auf Bildung- und Qualitätsfragen bei allen Formaten von Freiwilligendiensten. Ziel muss es hierbei sein, dass vergleichbare Standards für alle Freiwilligendienstformate gelten, sofern sie auf die gleichen Zielgruppen fokussieren.

Ausgangspunkt für alle Überlegungen ist aus Sicht der Evangelischen Trägergruppe neben dem Subsidiaritätsprinzip die konsequente Verankerung des Trä- gerprinzips in allen Formaten sowie die vollständige Übertragung der Verantwortung für die gesamte Bildungsarbeit auch im Bundesfreiwilligendienst.

Auf dem Hintergrund der 60-jährigen Geschichte des Diakonischen Jahr und dem vor drei Jahren begonnenen Paradigmenwechsel mit einem Ausbau der Freiwilligendienste hat die Diakonie Deutschland in ihrer Schriftenreihe "Auf den Punkt gebracht « unter dem Titel »Evangelische Freiwilligendienste 2020 « im Jubiläumsjahr 14 Thesen zu deren Weiterentwicklung vorgelegt (vgl. Kasten). Darin sind neben den hier genannten Wünschen und Erwartungen weitere Überlegungen und gesellschaftliche Notwendigkeiten beleuchtet und in kompakter Form zusammengefasst worden.

\section{Rechtliche Fragen der Selbstständigkeit}

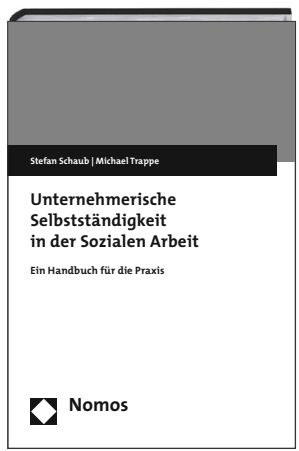

\section{Unternehmerische Selbstständigkeit in der Sozialen Arbeit}

Von Prof. Dr. Stefan Schaub und Dr. Michael Trappe

2014, ca. 392 S., brosch., ca. 72,- $€$ ISBN 978-3-8487-1269-4

Erscheint ca. November 2014 www.nomos-shop.de/22563

Alle Preise inkl. MwSt.

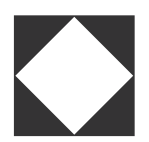

Nomos 
"So schnell der Hase der freiwilligen Tätigkeit auch rennt, der lgel Staat war immer schon da." Ralf Dahrendorf, deutscher Soziologe (1929-2009)

"Wer in der Demokratie schläft, erwacht in der Diktatur. " Hermann Glaser, deutscher Kulturpolitiker und Schriftsteller (geb. 1928)

"Aber die Armen sind auf die Gerechtigkeit angewiesen, die Reichen sind auf die Ungerechtigkeit angewiesen, das entscheidet. " Bertolt Brecht, deutscher Schriftsteller (1898-1956)

„Wenn eine freie Gesellschaft den vielen, die arm sind, nicht helfen kann, kann sie auch die wenigen nicht retten, die reich sind." John F. Kennedy, amerikanischer Politiker (1917-1963)

"Kuchen gibt es nie allein. Sondern immer nur zusammen mit einem Vortrag." Georg Schramm, Kabarettist, in seinem "Bericht aus einem Altenheim»

"Eine staatliche Reform lässt sich erzwingen, eine Hausreform aber ermöglicht sich nur auf dem Wege friedlicher Kompromisse. " Theodor Fontane, deutscher Schriftsteller (1819-1898)

"Keine Regierung und keine Bataillone vermögen Recht und Freiheit zu schützen, wo der Bürger nicht imstande ist, selber vor die Haustüre zu treten und nachzusehen, was es gibt. " Gottfried Keller, schweizerischer Schriftsteller (1819-1890) 